\title{
Cognitive functioning throughout the treatment history of clinical late-life depression
}

\author{
Joseph M. Dzierzewski ${ }^{1,2}$, Guy G. Potter ${ }^{3}$, Richard N. Jones ${ }^{4}$, Ola S. Rostant ${ }^{5}$, Brian Ayotte ${ }^{6}$, Frances M. Yang ${ }^{7}$, \\ Bonnie C. Sachs ${ }^{8}$, Betsy J. Feldman ${ }^{9}$ and David C. Steffens ${ }^{10}$ \\ ${ }^{1}$ David Geffen School of Medicine, UCLA, Los Angeles, CA, USA \\ ${ }^{2}$ Geriatric Research, Education and Clinical Center, Greater Los Angeles Veterans Affairs Healthcare System, Los Angeles, CA, USA \\ ${ }^{3}$ Department of Psychiatry and Behavioral Sciences, Duke University Medical Center, Durham, NC, USA \\ ${ }^{4}$ Department of Psychiatry and Human Behavior, Warren Alpert Medical School, Brown University, Providence, RI, USA \\ ${ }^{5}$ National Institute on Aging, Intramural Research Program, National Institutes of Health and Department of Psychiatry, University of \\ Michigan, Anne Arbor, MI, USA \\ ${ }^{6}$ Department of Psychology, University of Massachusetts Dartmouth, Dartmouth, MA, USA \\ ${ }^{7}$ Department of Biostatistics and Epidemiology, Medical College of Georgia, Georgia Regents University, Augusta, GA, USA \\ ${ }^{8}$ Department of Neurology, Wake Forest University Baptist Medical Center, Winston-Salem, NC, USA \\ ${ }^{9}$ Partners for Children, School of Social Work, University of Washington, Seattle, WA, USA \\ ${ }^{10}$ Department of Psychiatry, University of Connecticut, Farmington, CT, USA \\ Correspondence to: Joseph M. Dzierzewski, PhD, E-mail: Joseph.Dzierzewski@va.gov
}

Objective: Previous investigations into the relationship between late-life depressive symptoms and cognitive functioning have resulted in mixed findings concerning whether or not depressive symptoms and cognitive functioning are related. The mixed reports may be due in part to differences in clinical and nonclinical samples and to inadequate consideration of the dynamic nature (i.e., fluctuating course) of depressive symptoms and cognitive functioning in older adults. The current study examined the chronic, acute, and longitudinal relationships between depressive symptoms and cognitive functioning in older adults in an ongoing treatment study of major depressive disorder (MDD).

Methods: The neurocognitive outcomes of depression in the elderly study operates in a naturalistic treatment milieu using a pharmacological treatment algorithm and regular psychiatric assessment. Four hundred and fifty-three older adults [mean age 70 years, standard deviation $(S D)=7.2$ ] meeting criteria for MDD at study enrollment received annual neuropsychological testing and depressive symptom monitoring for an average of 8.5 years $(\mathrm{SD}=4.5)$.

Results: Hierarchical linear modeling revealed that higher age, lower education, and higher average/chronic levels of depressive symptoms were related to lower cognitive functioning. Additionally, results revealed that when an individual's depressive symptoms are higher than is typical for a specific individual, general cognitive function was worse than average. There was no evidence of lagged/longitudinal relationships between depressive symptoms and cognitive functioning in older adults in treatment for MDD.

Conclusions: Cognitive functioning and depressive symptoms are concurrently associated in older adults with MDD, highlighting the potential importance for stabilizing mood symptoms as a means to manage cognitive deficits in late-life depression. Copyright (C) 2015 John Wiley \& Sons, Ltd.

\begin{abstract}
Key words: major depressive disorder; cognitive functioning; depressive symptoms; naturalistic study; longitudinal design History: Received 23 September 2014; Accepted 6 January 2015; Published online 20 February 2015 in Wiley Online Library (wileyonlinelibrary.com)

DOI: 10.1002 /gps.4264
\end{abstract}

\section{Introduction}

The presence of both subclinical depressive symptoms and clinical depressive symptoms are quite common in older adults, with prevalence estimates reported at $9.8 \%$ and $2.3-4.8 \%$, respectively (Byers et al., 2010; Gum et al., 2009; Meeks et al., 2011). Depression is related to a number of negative outcomes 
among older adults, including increased rates of healthcare utilization (Feng et al., 2009) and costs (Katon et al., 2003), poor sleep (Buysse, 2004), caregiver burden (Morrow-Howell and Proctor, 1998), and increased mortality (Schulz et al., 2002). Decades of anecdotal clinical reports suggest that older adults with significantly elevated levels of depressive symptoms also experience decrements in cognitive functioning. Such clinical data have fueled empirical investigations into the relationships between late-life depressive symptoms and cognitive functioning; however, these investigations have not produced consistent results (e.g., Dotson et al., 2008; Han et al., 2006). Potential explanations for the disparate results include investigation of these relationships in nonclinical samples of older adults and the fluctuating course of both late-life cognitive functioning (Dzierzewski et al., 2013; Hultsch et al., 2002) and late-life depression (Beekman et al., 2002; Dautovich et al., 2014). The present investigation sought to investigate the relationships between depressive symptoms and cognitive functioning across the treatment history of late-life major depressive disorder (MDD).

Cross-sectional investigations examining the relationship between depressive symptoms and cognitive functioning in late-life have consistently reported a significant negative relationship between depressive symptoms and cognitive functioning (Dotson et al., 2008; Ganguli et al., 2006; Lichtenberg et al., 1995; Vinkers et al., 2004). However, the longitudinal relationship between depressive symptoms and cognitive functioning has not been observed as consistently. Several longitudinal examinations indicate that initial levels of depressive symptoms are unrelated to longitudinal decline in cognitive functioning in older adults (Ganguli et al., 2006; Han et al., 2006), whereas other investigators report that depressive symptoms are related to subsequent cognitive decline (Dotson et al., 2008; van den Kommer et al., 2013; Wilson et al., 2004). Notably, participants in the aforementioned studies were generally not selected for study inclusion on the basis of their depressive symptoms.

Alternatively, some investigators have suggested that cognitive functioning may be a prodromal predictor of subsequent changes in depressive symptoms. It has been reported that older adults with worse baseline cognitive functioning had an accelerated increase of depressive symptoms; however, individuals with worse depressive symptoms at baseline did not have an acceleration in cognition decline (Jajodia and Borders, 2011; Perrino et al., 2008; Vinkers et al., 2004). As these studies did not select their samples based on clinical diagnosis of depressive symptoms, authors have speculated that "individuals with stronger clinical histories might experience a different process" (Jajodia \& Borders, 2011).

In addition to the distinction between clinical and subclinical depressive symptoms, some of the disparate findings regarding the relationship between depressive symptoms and cognitive functioning may be in part due to the fluctuating nature of both late-life depression and late-life cognitive functioning. Depressive disorders in older adults are characterized by "prolonged fluctuating symptoms" (i.e., variability among individual symptoms over time) (Beekman et al., 2002) with a "fluctuating course" (i.e., changes in the overall symptom severity) (Sanders et al., 2011). Dautovich, Dzierzewski, and Gum (2014) reported that among older adults with clinically relevant levels of depression, depressive symptoms fluctuated greatly from observation to observation (Dautovich et al., 2014). Likewise, the cognitive functioning (as operationalized by cognitive test performance) of nondepressed, community-dwelling older adults also fluctuates greatly from occasion to occasion (Dzierzewski et al., 2013; Hultsch et al., 2002), with the amount of fluctuation often being reported as an important predictor of cognitive plasticity, dementia status, and even death (Dzierzewski et al., 2013; Hultsch et al., 2000; MacDonald et al., 2008). Previous research into the fluctuating nature of both depressive symptoms and cognitive functioning suggests that concomitant fluctuations in depressive symptoms may contribute to the variability of cognitive performance over time. In fact, it has been reported that at an assessment when depressive symptoms worsened, general cognitive functioning also worsened (Han et al., 2006). However, like other studies into the relationship between depressive symptoms and cognitive functioning, the sample was not selected on the basis of the presence of clinically significant depressive symptoms.

The referenced studies portray a rather complex relationship between late-life depressive symptoms and cognitive functioning, with questions remaining regarding the directionality of the relationships and whether previous findings in nonclinical samples may generalize to clinically depressed older adults under treatment. We sought to address these questions by examining the relationships between depressive symptoms and cognitive functioning in a sample of older adults under long-term treatment for MDD. Specific aims of the investigation were the following: (i) to examine the bidirectional relationships between chronic depressive symptoms and cognitive 
functioning in older adults under treatment for MDD; (ii) to examine the bidirectional relationships between concurrent intrapersonal fluctuations in depressive symptoms and intrapersonal changes in cognitive functioning in older adults with MDD; and (iii) to examine the bidirectional cross-lagged relationships between depressive symptoms and subsequent cognitive functioning in older adults under treatment for MDD. We hypothesized the following: (i) depressive symptoms and cognitive functioning would be chronically related; (ii) depressive symptoms and cognitive functioning would be acutely related; and (iii) there would be a lagged relationship (1-year longitudinal) between depressive symptoms and subsequent cognitive functioning (but not vice versa) in older adults under treatment for MDD.

\section{Methods}

\section{Participants}

All participants were enrolled in the National Institute of Mental Health-sponsored Mental Health Clinical Research Center, neurocognitive outcomes of depression in the elderly (NCODE) study at Duke University Medical Center (Steffens et al., 2004). Participants were 60 years or older with a diagnosis of MDD at the time of enrollment. Exclusion criteria at the time of enrollment included the following: (i) another major psychiatric illness (bipolar disorder, schizophrenia, and dementia); (ii) alcohol/drug abuse or dependence; and (iii) primary neurologic illness. All individuals were screened for vision, hearing, and motor limitations that may interfere with valid neuropsychological test administration, and no participants were excluded on the basis of screening results. Individuals were screened for dementia at time of enrollment on the basis of an established study protocol that included review of a comprehensive clinical evaluation, consultation with referring physicians, and cognitive screening with the mini-mental state examination (MMSE; Folstein et al., 1975). Clinicians were allowed to exclude participants if clinical or medical history was suspicious for cognitive decline; however, none of the participants were excluded because of suspected dementia. Additionally, individuals performing $<25$ on the MMSE were reassessed after an 8-week phase of treatment and subsequently included for longitudinal follow-up if their MMSE score was $\geq 25$ at that time. No individuals were excluded because of low MMSE. NCODE was approved by the Duke University Institutional Review Board.

\section{Design and measures}

At the time of study enrollment, a geriatric psychiatrist interviewed each participant and assessed depression symptoms with a number of standardized clinical assessments (Steffens et al., 2004). The clinical interview included the 10-item Montgomery-Asberg Depression Rating Scale (MADRS; Montgomery and Asberg, 1979). The MADRS has a range of 0-60 (higher scores representing more depressive symptoms) and was repeated at annual assessments. Scores on the MADRS $>15$ indicate clinically meaningful levels of depressive symptoms, with scores $>35$ indicating severe depression. The MADRS has demonstrated good psychometric properties within the NCODE study (Steffens et al., 2004) and has been validated for use in geriatric patients (Engedal et al., 2012). The NCODE study operates in a naturalistic treatment milieu using treatment guidelines established by the Duke Affective Disorders Program (Steffens et al., 2002). The majority of individuals were treated with a selective serotoninreuptake inhibitor as a first-line treatment, with changes in treatment procedures based on the treatment response and the Duke Somatic Treatment Algorithm for Geriatric Depression (Steffens et al., 2002). The protocol recommends that patients receive continuation treatment for at least 1-2 years (some indefinitely) once they achieve remission. Neuropsychological assessment occurred at study enrollment and was repeated annually. The assessment included the Consortium to Establish a Registry for Alzheimer's Disease (CERAD) neuropsychological battery (Morris et al., 1989). The current study used the CERAD Total Score (CERAD-TS; Chandler et al., 2005), as an index of global neurocognitive functioning. The CERAD-TS includes measures of immediate memory, delayed memory, recognition memory, semantic fluency, object naming, and constructional praxis, with a score ranging from 0 to 100 with higher scores indicating better cognitive functioning. The complete study design, including assessment of depressive symptoms and neuropsychological assessment have been described in detail elsewhere (Steffens et al., 2004). The current study utilized the annual CERAD-TS and MADRS scores.

\section{Statistical analysis}

Data were analyzed with IBM SPSS 22 statistical software. Statistical analysis addressed the bidirectional relationship between chronic depressive symptoms and cognitive functioning in older adults 
under treatment for MDD (aim 1), the bidirectional relationship between concurrent (i.e., acute) intrapersonal fluctuations in depressive symptoms and intrapersonal changes in cognitive functioning in older adults with MDD (aim 2), and the bidirectional 1-year lagged (i.e., longitudinal) relationship between depressive symptoms and subsequent cognitive functioning in older adults under treatment for MDD (aim 3). The first step was to determine whether the current clinically depressed sample displayed sufficient fluctuation in cognitive functioning and depressive symptoms to justify the examination of dynamic relationships between depression and cognition. This was accomplished through implementation of a predictor-free hierarchical linear model (HLM; Singer and Willett, 2003) and calculation of the intraclass correlation coefficient [ICC: $\sigma^{2}$ (between)/ $\sigma^{2}$ (between + within)].

To accomplish the primary study aims, depressive symptoms (i.e., MADRS scores) were used to predict cognitive functioning (i.e., CERAD-TS) within an HLM framework. In order to examine the direction of the depressive symptoms-cognitive functioning relationship, we also used cognitive functioning (i.e., CERADTS) to predict depressive symptoms (i.e., MADRS scores). Such an approach provided an opportunity to examine how well depressive symptoms predicted cognitive functioning (and vice versa) both concurrently and 1-year lagged within-persons (level 1) and between-persons (level 2). Level 2 submodels examined questions such as: Do people who generally exhibit more depressive symptoms (i.e., higher chronic depression) also experience lower levels of cognitive functioning? Concurrent level 1 submodels addressed questions related to acute changes, such as: When a person exhibits depressive symptoms above their personal average, does he or she also experience relative decreased cognitive functioning? Lagged level 1 submodels addressed longitudinal questions such as: $A$ year after an assessment when a person exhibits above-average depressive symptoms, does he or she experience a subsequent decrease in cognitive functioning? As such, this study sought to address the chronic, acute, and longitudinal associations among depressive symptoms and cognitive functioning (and vice versa). Participant attrition and missing data are common problems in longitudinal designs. However, participants with missing data are not excluded from analyses within an HLM framework, as all available data are utilized in analyses via maximum likelihood estimates. All models controlled for the following covariates: linear and quadratic time trends, age, education level, sex, and age $\times$ time trends interactions.
In follow-up analyses, the aforementioned models predicting cognitive functioning were rerun to explore the possibility that baseline depressive symptoms may be overinfluencing the model. All participants carried a diagnosis of MDD upon entry to the study. As the study design was a naturalistic treatment milieu, study participants received treatment aimed at reducing depressive symptoms. As such, depressive symptomology was expected to be highest at study entry, which could result in undue model influence. To test this idea, all models were reparameterized removing baseline values of the MADRS and examined for change in model estimates.

\section{Results}

\section{Descriptive statistics}

Data from 453 older adults were analyzed. Participants had an average age of 70 years (standard deviation $(\mathrm{SD})=7.2$ ] at study enrollment, completed 14 years of schooling, $(\mathrm{SD}=3.0)$, experienced a mild level of depressive symptoms on average throughout their time in the study (mean MADRS $14 \pm 8.5$ ), and were enrolled in the study for an average of 8.5 years $(\mathrm{SD}=4.5)$. Study participants provided an average of 5.6 data points (range 1-13). The mean cognitive functioning (CERAD-TS across time and participants) was $74(\mathrm{SD}=12.3)($ Table 1$)$.

\section{Fluctuations in cognition and depressive symptoms}

The ICC was 0.78 for CERAD-TS, indicating that $22 \%$ of the overall variance in cognitive functioning was a within-person (i.e., over time) phenomenon. The

Table 1 Demographic and clinical data $(n=453)$

\begin{tabular}{lc}
\hline & Mean (SD) \\
\hline Age & $70(7.2)$ \\
Education & $14(3.0)$ \\
Number of visits & $5.6(2.9)$ \\
Years in study & $8.5(4.5)$ \\
Sex (\% female) & 68 \\
Race (\% White) & 85 \\
Marital status (\% married) & 59 \\
MADRS (initial) & $23(6.8)$ \\
CERAD-TS (initial) & $76(12)$ \\
MADRS (average) & $14(8.5)$ \\
CERAD-TS (average) & $74(12.3)$ \\
\hline
\end{tabular}

SD, standard deviation; MADRS, Montgomery-Asberg Depression Rating Scale; CERAD-TS, Consortium to Establish a Registry for Alzheimer's Disease neuropsychological battery, Total Score. 
ICC was 0.33 for MADRS, indicating that $67 \%$ of the overall variance in depressive symptoms was a within-person phenomenon. See Figure 1 for a graphical representation of individual CERAD-TS and MADRS across the study period. As illustrated in Figure 1, an individual's unique cognitive functioning and depressive symptoms varied widely from assessment to assessment, suggesting that our conceptual and analytic approach was appropriate.

\section{Depressive symptoms predicting cognitive functioning}

In the HLM predicting concurrent cognitive functioning, average level of depressive symptoms $[B=-0.37, t(294)=-3.77, p<0.001]$ was a significant between-person (level 2) predictor, as was age $[B=-0.31, t(484)=-3.03, p<0.01]$ and education $[B=1.54, t(285)=6.65, p<0.001]$. Older adults with higher levels of chronic depressive symptoms had lower-than-average overall cognitive functioning. At the within-person level (level 1), person-centered depressive symptoms $[B=-0.11, \quad t(152)=-3.41$, $p<0.01]$ was a significant predictor of cognitive function. This model revealed an acute relationship; when an individual exhibited depressive symptoms that are above their intrapersonal average, they also displayed worse cognitive functioning than average. Other level 1 significant predictors included quadratic time $[B=0.51, t(163)=2.14, p<0.05]$ and the age $\times$ quadratic time interaction $[B=-0.01, t(167)=-2.23, p<0.05]$, suggesting that older participants demonstrated accelerated decline in cognitive functioning. The model explained $43 \%$ of the within-person variance and $40 \%$ of the between-person variance in cognitive functioning. The lagged model was not suggestive of a longitudinal relationship. Depressive symptoms do not predict cognitive functioning 1 year later in older adults under treatment for MDD (Table 2).

In follow-up analysis examining the potential overinfluence of baseline depressive symptoms, the model resulted in an identical pattern of results and significance, indicating no undue influence of baseline level of depressive symptoms on the model observed relationships between depressive symptoms and cognitive functioning.

\section{Cognitive functioning predicting depressive symptoms}

The final HLM predicting concurrent depressive symptoms revealed a similar pattern of results as those for the model predicting cognitive functioning. Older adults and individuals with better average cognitive functioning had lower than average overall chronic depressive symptoms. Additionally, older individuals exhibited accelerated decline in depressive symptoms, and when an individual exhibited acute cognitive functioning above their intrapersonal average, they also displayed acute lower depressive symptoms than average. Again, the lagged model did not suggest that cognitive functioning predicted 1-year longitudinal change in depressive symptoms in older adults under treatment for MDD (Table 3).
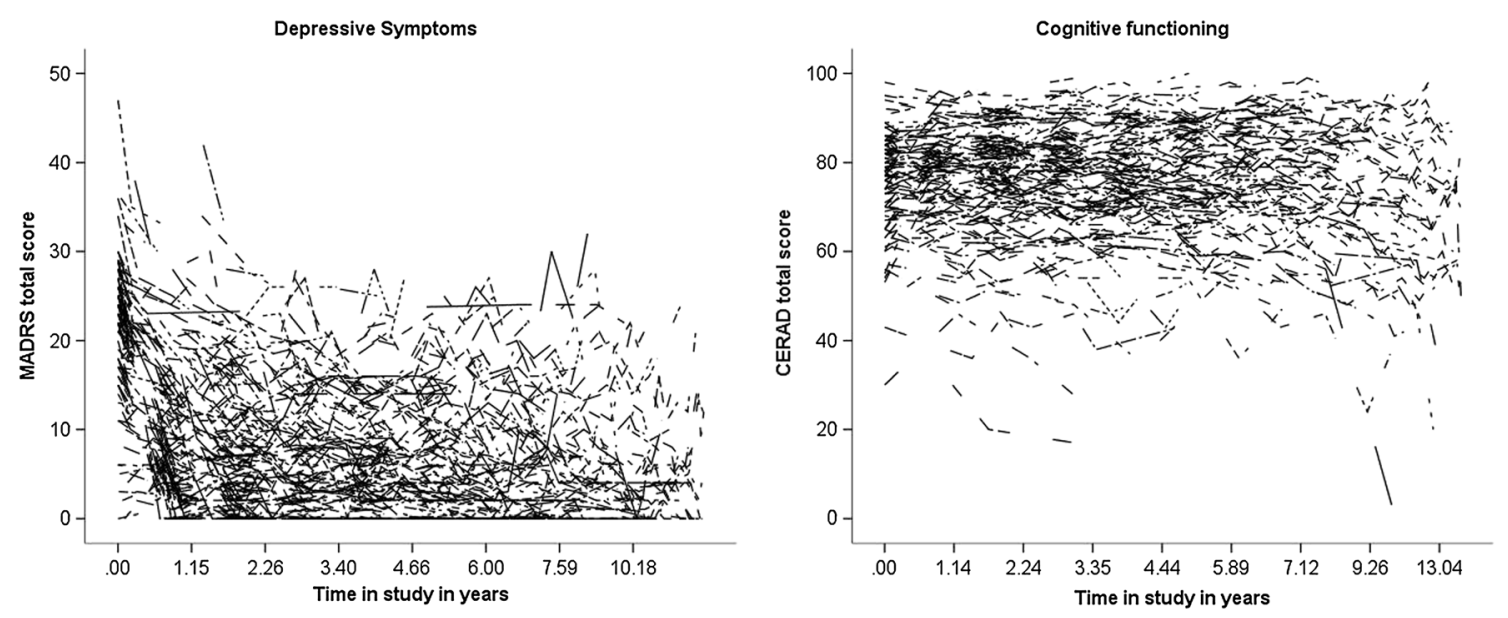

Figure 1 Graph showing within-person fluctuations in cognitive functioning [Consortium to Establish a Registry for Alzheimer's Disease neuropsychological battery, Total Score (CERAD-TS), right panel] and depressive symptoms [Montgomery-Asberg Depression Rating Scale (MADRS), left panel] across study period. Each individual's data is represented by a single line. 
Table 2 Multilevel Models Predicting Cognitive Functioning from Depression.

\begin{tabular}{|c|c|c|c|c|c|c|c|c|}
\hline \multirow[b]{3}{*}{ Predictor Variable } & \multicolumn{4}{|c|}{ Concurrent Model } & \multicolumn{4}{|c|}{ Lagged Model } \\
\hline & \multicolumn{8}{|c|}{ Fixed Effects } \\
\hline & $B$ & $S E$ & $d f$ & $t$ & $B$ & SE & $d f$ & $t$ \\
\hline \multicolumn{9}{|l|}{ Within-person (Level 1) } \\
\hline Linear time & 2.45 & 2.24 & 845.67 & 1.09 & 13.56 & 6.69 & 179.49 & $2.03^{*}$ \\
\hline Quadratic time & 0.51 & 0.24 & 163.42 & $2.14^{\star}$ & -0.67 & 0.62 & 116.86 & -1.08 \\
\hline Age $\times$ Linear time & -0.03 & 0.03 & 854.69 & -1.02 & -0.19 & 0.09 & 184.60 & $-2.01^{*}$ \\
\hline Age $\times$ Quadratic & -0.01 & 0.003 & 167.38 & $-2.32^{*}$ & 0.01 & 0.01 & 119.47 & 1.08 \\
\hline Depression & -0.11 & 0.03 & 152.13 & $-3.41^{\star \star}$ & 0.02 & 0.10 & 23.55 & 0.19 \\
\hline \multicolumn{9}{|l|}{ Between-person (Level 2) } \\
\hline Age & -0.31 & 0.10 & 484.10 & $-3.03^{\star \star}$ & -0.07 & 0.24 & 317.83 & -0.28 \\
\hline Sex & 0.93 & 1.26 & 273.00 & 0.74 & 1.50 & 1.81 & 80.21 & 0.83 \\
\hline Education & 1.54 & 0.23 & 284.66 & $6.65^{\star \star \star}$ & 1.34 & 0.39 & 96.10 & $3.43^{\star \star \star}$ \\
\hline \multirow[t]{2}{*}{ Depression } & -0.37 & 0.10 & 293.53 & $-3.77^{\star \star \star}$ & -0.40 & 0.17 & 79.80 & $-2.31^{\star}$ \\
\hline & \multicolumn{8}{|c|}{ Random Effects } \\
\hline Covariance parameter estimate & $B$ & SE & & $Z$ & $B$ & SE & & $Z$ \\
\hline \multicolumn{9}{|l|}{ Within-person } \\
\hline Linear time & .85 & .27 & & $3.17^{\star *}$ & .22 & .21 & & 1.03 \\
\hline Quadratic time & .003 & .002 & & 1.55 & $.000^{\mathrm{a}}$ & $.000^{\mathrm{a}}$ & & --- \\
\hline \multirow[t]{3}{*}{ Depression } & .04 & .02 & & $2.38^{*}$ & .23 & .18 & & 1.30 \\
\hline & & \multirow{2}{*}{\multicolumn{2}{|c|}{$\begin{array}{l}\text { Within Pseudo } R^{2} \\
\text { Between Pseudo } R^{2}\end{array}$}} & $43 \%$ & & \multirow{2}{*}{\multicolumn{2}{|c|}{$\begin{array}{l}\text { Within Pseudo } R^{2} \\
\text { Between Pseudo } R^{2}\end{array}$}} & $50 \%$ \\
\hline & & & & $40 \%$ & & & & $49 \%$ \\
\hline
\end{tabular}

Note: Cognitive functioning is operationalized as cognitive test performance. Between-person depression refers to person-mean level of the MADRS (i.e., average level of depression over the entire study period); In the Concurrent Model within-person depression refers to person-centered level of the MADRS (i.e., annual deviations in depression centered around person-mean level of depression); In the Lagged Model within-person depression refers to lagged person-centered level of the MADRS (i.e., previous year annual deviations in depression centered around person-mean level of depression).

${ }^{a}$ Variance too small to be estimated. The final Hessian matrix is not positive definite although all convergence criteria are satisfied. The test statistic and confidence interval cannot be computed.

*** Predictor is significant at .001 level;

** Predictor is significant at the .01 level;

* Predictor is significant at the .05 level.

\section{Discussion}

Consistent with previous research (Dzierzewski et al., 2013; Hultsch et al., 2002), we observed large fluctuations in global cognitive functioning (i.e., $22 \%$ of the total variance in cognitive functioning) in a group of older adults under long-term treatment for depression. We also replicated previous findings of increased age and lower educational attainment being related to lower global cognitive functioning (Plassman et al., 1995). Consistent with our first hypothesis, we found that higher levels of chronic depressive symptoms are related to lower global cognitive functioning (Dotson et al., 2008; Ganguli et al., 2006; Lichtenberg et al., 1995; Vinkers et al., 2004). In support to our second hypothesis and unique to the current investigation, we discovered that acute fluctuations in depressive symptomology were associated with fluctuations in global cognitive functioning (and vice versa) in older adults under treatment for MDD. Our third hypothesis was not supported. We did not observe a 1-year longitudinal relationship between depressive symptoms and cognitive functioning (or vice versa) in older adults in treatment for MDD. Stated differently, in our sample of clinically depressed older adults, the course of depressive symptoms and cognitive functions appear to be tightly temporally related (i.e., when an individual experiences depressive symptoms above their own personal average, they experience cognitive functioning below their own personal average). Neither depressive symptoms nor cognitive functioning at one occasion predicted subsequent level of functioning 1 year later.

The finding that higher chronic levels of depressive symptoms are associated with lower levels of global cognitive functioning adds to a growing literature that recognizes the importance of depression on late-life cognitive functioning (Dotson et al., 2008; Han et al., 2006; Wilson et al., 2004). Importantly, our results were found in a sample of older adults with formal 
Table 3 Multilevel models predicting depression from cognitive functioning

\begin{tabular}{|c|c|c|c|c|c|c|c|c|}
\hline \multirow[b]{3}{*}{ Predictor variable } & \multicolumn{4}{|c|}{ Concurrent model } & \multicolumn{4}{|c|}{ Lagged model } \\
\hline & \multicolumn{8}{|c|}{ Fixed effects } \\
\hline & $B$ & SE & $d f$ & $t$ & $B$ & SE & $d f$ & $t$ \\
\hline \multicolumn{9}{|l|}{ Within-person (Level 1) } \\
\hline Linear time & -13.62 & 2.14 & 551.59 & $-6.35^{\star \star \star}$ & 8.89 & 5.56 & 143.76 & 1.60 \\
\hline Quadratic time & 1.30 & 0.21 & 95.71 & $6.24^{\star \star \star}$ & -0.63 & 0.53 & 63.31 & -1.17 \\
\hline Age $\times$ Linear time & 0.16 & 0.03 & 579.78 & $5.20^{\star \star *}$ & -0.12 & 0.08 & 155.10 & -1.56 \\
\hline Age $\times$ Quadratic & -0.02 & 0.003 & 99.86 & $-5.61^{\star \star *}$ & 0.01 & 0.01 & 67.33 & 1.13 \\
\hline Cognitive functioning & -0.14 & 0.04 & 123.27 & $-3.10^{\star \star}$ & 0.01 & 0.07 & 236.49 & 0.17 \\
\hline \multicolumn{9}{|l|}{ Between-person (Level 2) } \\
\hline Age & -0.36 & 0.08 & 581.16 & $-4.44^{\star \star \star}$ & 0.23 & 0.19 & 310.62 & 1.22 \\
\hline Sex & 0.98 & 0.77 & 210.99 & 1.27 & 1.74 & 1.08 & 110.84 & 1.61 \\
\hline Education & -0.05 & 0.15 & 236.04 & -0.32 & -0.01 & 0.25 & 125.42 & -0.04 \\
\hline \multirow[t]{2}{*}{ Cognitive functioning } & -0.14 & 0.04 & 228.17 & $-3.97^{\star \star \star}$ & -0.09 & 0.05 & 142.42 & -1.72 \\
\hline & \multicolumn{8}{|c|}{ Random effects } \\
\hline Covariance parameter estimate & $B$ & SE & & Z & $B$ & SE & & $Z$ \\
\hline \multicolumn{9}{|l|}{ Within-person } \\
\hline Linear time & 0.08 & 0.10 & & 0.76 & 0.03 & 0.10 & & 0.29 \\
\hline Quadratic time & 0.0004 & 0.001 & & 0.47 & $0.000^{\mathrm{a}}$ & $0.000^{\circ}$ & & - \\
\hline \multirow[t]{2}{*}{ Cognitive functioning } & 0.08 & 0.03 & & $2.52^{*}$ & $0.000^{\mathrm{a}}$ & $0.000^{2}$ & & - \\
\hline & & \multicolumn{2}{|c|}{$\begin{array}{l}\text { Within pseudo } R^{2} \\
\text { Between pseudo } R^{2}\end{array}$} & $\begin{array}{l}33 \% \\
2 \%\end{array}$ & & \multicolumn{2}{|c|}{$\begin{array}{l}\text { Within pseudo } R^{2} \\
\text { Between pseudo } R^{2}\end{array}$} & $\begin{array}{c}59 \% \\
9 \%\end{array}$ \\
\hline
\end{tabular}

Cognitive functioning is operationalized as cognitive test performance. Between-person cognitive function refers to person-mean level of the CERAD neuropsychological battery (i.e., average level of cognitive function over the entire study period). In the concurrent model, within-person cognitive function refers to person-centered level of CERAD neuropsychological battery (i.e., annual deviations in cognitive function centered around personmean level of cognitive function). In the lagged model, within-person cognitive function refers to lagged person-centered level of the CERAD neuropsychological battery (i.e., previous year annual deviations in cognitive function centered around person-mean level of cognitive function). ${ }^{a}$ Variance too small to be estimated. The final Hessian matrix is not positive definite although all convergence criteria are satisfied. The test statistic and confidence interval cannot be computed.

*** Predictor is significant at 0.001 level;

${ }^{* *}$ Predictor is significant at the 0.01 level;

*Predictor is significant at the 0.05 level.

diagnosis and treatment for MDD, which extends previous relationships found in preclinical mood disorders.

Previous longitudinal examinations report that baseline depressive symptoms predict negative changes in late-life cognitive functioning (Dotson et al., 2008; van den Kommer et al., 2013; Wilson et al., 2004) and that baseline cognitive functioning predicts negative changes in late-life depressive symptoms (Jajodia \& Borders, 2011; Perrino et al., 2008; van den Kommer et al., 2013; Vinkers et al., 2004). In the present study, we did not observe this longitudinal relationship, in either direction. A distinction between the present sample and those analyzed in other investigations is that our sample met criteria for MDD at baseline and subsequently underwent treatment for MDD. Perhaps the relationship between depressive symptoms and cognitive functioning is distinct between subclinical and clinical depressive symptoms and may differ when older adults are actively engaged in treatment and potentially transition from clinically depressed to not depressed. Future research should explore this possibility.

Previous investigations did not actively account for the fluctuating nature of late-life depressive symptoms. Late-life depressive symptoms are not static; in fact, late-life depression displays substantial changes across time within older adults (Beekman et al., 2002; Dautovich et al., 2014). In this case, we found that deviations around an older adult's average level of depressive symptoms was associated with fluctuations in their global cognitive functioning even when participants were actively engaged in treatment for their depression. In other words, acute changes in late-life depressive symptoms are associated with changes in late-life global cognitive functioning in older adults diagnosed and receiving treatment for MDD. Such a finding contributes to our understanding that late-life MDD is a cognitive disorder and a mood disorder, and their etiology and pathogenesis may be linked. 
There are several clinical implications of the current investigation. Mood and cognition are dynamically related concurrently in depressed older adults in treatment for MDD. As such, older adults with comorbid late-life depression and cognitive difficulties should engage in pharmacotherapy and/or psychotherapy in an attempt not only to improve their affective state but also to indirectly improve their immediate cognitive functioning. Research suggests that medication treatment of depressive symptoms in late-life can have a positive impact on cognitive functioning (Butters et al., 2000; Doraiswamy et al., 2003); however, the effects of psychotherapy for late-life depression on cognitive functioning remains underexplored.

The concomitant hypothesis of depression and cognition in late-life posits that a common factor may be causing both the depressive symptoms and cognitive dysfunction. As we identified concurrent associations between depressive symptoms and cognitive functioning in older adults with MDD, our results provide support for the concomitant hypothesis. We observed neither a lagged relationship from depressive symptoms to subsequent cognitive functioning or from cognitive functioning to subsequent depressive symptoms. Such data suggest that the focus of treatment be on immediate and current improvements in functioning as opposed to long-term prevention.

Some limitations of the current study should be noted. First, data were gathered from depressed older adults whom all were actively engaged in treatment, and individuals were treated with several different psychotropic medications and combinations of medications. Most individuals were treated with a selective serotonin-reuptake inhibitor as a first-line treatment, with changes in treatment procedures based on the treatment response and the Duke Somatic Treatment Algorithm for Geriatric Depression (Steffens et al., 2002). We are limited in our ability to analyze potential effects of single antidepressant agents over individual longitudinal courses of treatment; however, evidence suggests most antidepressant medications have small-to-nonexistent direct effects on neurocognitive performance (Podewils and Lyketsos, 2002; Siepmann et al., 2003). Second, while we did observe relationships between our variables of interest from year to year, perhaps annual measurement is not the ideal time frame to study the link between mood and cognitive functioning in late-life. Future studies should collect data in multiple time samples in order to more finely model the relationships between mood and cognitive functioning. Third, the CERAD-TS lacks measures of processing speed, which tends to persist with depression remission. It would be useful to track dynamic changes in processing speed and depression symptoms using an analogous modeling approach. Fourth, the presence of anxiety disorders, which often are comorbid with MDD, and other medical conditions/lifestyle factors with known relationships between mood symptoms and cognitive functioning were not accounted for within the current investigation. Lastly, generalizability may be limited by the highly educated nature of our clinical sample.

We discovered both a chronic and dynamic concurrent relationship between depressive symptoms and cognitive functioning (and vice versa) in older adults undergoing treatment for late-life MDD. The dynamic association between mood disturbance and late-life cognitive functioning suggests that above and beyond any cumulative effects of chronic mood on cognitive functioning in late-life, relatively short-term changes in mood can still impact cognitive functioning. Such a finding speaks importantly to the potential plasticity and resiliency of cognition in older adults who have experienced depression in later life, as well as to the importance of mental wellness for cognitive functioning in older adults.

\section{Conflict of interest}

None declared.

Key points

- In older adults receiving treatment for major depressive disorder, depressive symptoms and cognitive functioning are related such that when an individual experiences fluctuations in their mood, they also experience concurrent fluctuations in their cognitive functioning.

- In older adults receiving treatment for major depressive disorder, depressive symptoms do not predict later cognitive functioning, or vice versa.

\section{Acknowledgements}

This work was supported by a conference grant (R13 AG030995). The source study was supported by NIMH (MH54846). Joseph M. Dzierzewski was supported by UCLA Claude Pepper Center (5P30AG028748) and UCLA CTSI (UL1TR000124), and VA Advanced Geriatrics Fellowship. 


\section{References}

Beekman ATF, Geerlings SW, Deeg DJH, et al. 2002. The natural history of late-life depression: a 6-year prospective study in the community. Arch Gen Psychiatry 59: 605-611.

Butters MA, Becker JT, Nebes RD, et al. 2000. Changes in cognitive functioning following treatment of late-life depression. Am J Psychiatry 157: 1954.

Buysse DJ. 2004. Insomnia, depression, and aging: assessing sleep and mood interactions in older adultsv. Geriatrics 59: 47-51.

Byers AL, Yaffe K, Covinsky KE, Friedman MB, Bruce ML. 2010. High occurrence of mood and anxiety disorders among older adults: the national comorbidity survey replication. Arch Gen Psychiatry 67: 489-496.

Chandler MJ, Lacritz LH, Hynan LS, et al. 2005. A total score for the CERAD neuropsychological battery. Neurology 65: 102-106.

Dautovich ND, Dzierzewski JM, Gum AM. 2014. Older adults display concurrent but not delayed associations between life stressors and depressive symptoms: a microlongitudinal study. Am J Public Health, in press.

Doraiswamy PM, Krishnan KRR, Oxamn T, et al. 2003. Does antidepressant therapy improve cognition in elderly depressed patients? Journal of Gerontology: Medical Sciences 58A: 1137-1144.

Dotson VM, Resnick SM, Zonderman AB. Differential association of concurrent baseline, and average depressive symptoms with cognitive decline in older adults. The American journal of geriatric psychiatry: official journal of the American Association for Geriatric Psychiatry 16: 318-330. 4-1-2008.

Dzierzewski JM, Marsiske MM, Mrogan AA, et al. 2013. Cognitive inconsistency and practice-related learning in older adults. GeroPsych 26: 173-184.

Engedal K, Kvaal K, Korsnes M, et al. 2012. The validity of the Montgomery-Asberg Depression Rating Scale as a screening tool for depression in later life. J Affect Disord 141: 227-232.

Feng L, Yap KB, Kua EH, Ng TP. 2009. Depressive symptoms, physician visits and hospitalization among community-dwelling older adults. International Psychgeriatrics 21: 568-575.

Folstein MF, Folstein SE, McHugh PR. 1975. Mini-mental state: a practical method for grading the cognitive state of patients for the clinician. J Psychiatr Res 12. 189-198.

Ganguli M, Du Y, Dodge HH, Ratcliff GG, Chang CH. 2006. Depressive symptoms and cognitive decline in late life: a prospective epidemiological study. Arch Gen Psychiatry 63: 153-160.

Gum AM, King-Kalimanis B, Kohn R. 2009. Prevalence of mood, anxiety, and substance-abuse disorders for older Americans in the national comorbidity survey-replication. Am J Geriatr Psychiatry 17: 769-781.

Han L, McCusker J, Abrahamowicz M, Cole M, Capek R. 2006. The temporal relationship between depression symptoms and cognitive functioning in older medical patients: prospective or concurrent? J Gerontol A Biol Sci Med Sci 61: 1319-1323.

Hultsch DF, MacDonald SWS, Dixon RA. 2002. Variability in reaction time performance of younger and older adults. J Gerontol B Psychol Sci Soc Sci 57: 101-115.

Hultsch DF, MacDonald SWS, Hunter MA, Levy-Bencheton J, Strauss E. 2000. Intraindividual variability in cognitive performance in older adults: comparison of adults with mild dementia, adults with arthritis, and healthy adults. Neuropsychology 14: 588-598.
Jajodia A, Borders A. 2011. Memory predicts changes in depressive symptoms in older adults: a bidirectional longitudinal analysis. Journal of Gerontology: Psychological Sciences 66: 571-581.

Katon WJ, Lin E, Russo J, Unutzer J. 2003. Increased medical costs of a populationbased sample of depressed elderly patients. Arch Gen Psychiatry 60: 897-903.

Lichtenberg PA, Ross T, Millis SR, Manning CA. 1995. The relationship between depression and cognition in older adults: a cross-validation study. Journal of Gerontology: Psychological Sciences 50B: 25-32.

MacDonald SWS, Hultsch DF, Dixon RA. 2008. Predicting impending death: inconsistency in speed is a selective and early marker. Psychol Aging 23: 595-607.

Meeks TW, Vahia IV, Lavretsky H, Kulkarni G, Jeste DV. 2011. A tune in "a minor" can "b major": a review of epidemiology, illness course, and public health implications of subthreshold depression in older adults. J Affect Disord 129: 126-142.

Montgomery SA, Asberg M. 1979. A new depression scale designed to be sensitive to change. British Journal of Psychiatry 134: 382-389.

Morris JC, Heyman A, Mohs RC, et al. 1989. The Consortium to Establish a Registry for Alzheimer's Disease (CERAD). Part I. Clinical and neuropsychological assessment of Alzheimer's disease. Neurology 39: 1159-1165.

Morrow-Howell N, Proctor E. 1998. Informal caregiving to older adults hospitalized for depression. Aging Ment Health 2: 222-231.

Perrino T, Mason CA, Brown SC, Spokane A, Szapocznik J. 2008. Longitudinal relationships between cognitive functioning and depressive symptoms among Hispanic older adults. J Gerontol B Psychol Sci Soc Sci 63: 309-317.

Plassman BL, Welsh KA, Helms M, et al. 1995. Intelligence and education as predictors of cognitive state in late life. Neurology 45: 1446-1450.

Podewils LJ, Lyketsos CG. 2002. Tricyclic antidepressants and cognitive decline. Psychosomatics 43: 31-35.

Sanders JB, Bremmer MA, Comijs HC, et al. Cognitive functioning and the natural course of depressive symptoms in late life. The American journal of geriatric psychiatry: official journal of the American Association for Geriatric Psychiatry 19: 664-672. 7-1-2011.

Schulz R, Drayer RA, Rollman BL. 2002. Depression as a risk factor for non-suicide mortality in the elderly. Biol Psychiatry 52: 205-225.

Siepmann M, Grossmann J, Muck-Weymann M, Kirch W. 2003. Effects of sertraline on autonomic and cognitive functions in healthy volunteers. Psychopharmacology (Berl) 168: 293-298.

Singer, JD. Willett, J.B. 2003. Applied longitudinal data analysis: methods for studying change and event occurrence. Oxford University Press: New York.

Steffens DC, McQuoid DR, Krishnan KR. 2002. The Duke Somatic Treatment Algorithm for Geriatric Depression (STAGED) approach. Psychopharmacol Bull 36: $58-68$.

Steffens DC, Welsh-Bohmer KA, Burker JR, et al. 2004. Methodology and preliminary results from the neurocognitive outcomes of depression in the elderly study. $J$ Geriatr Psychiatry Neurol 17: 202-211.

van den Kommer TN, Comijs HC, Aartsen MJ, et al. 2013. Depression and cognition: how do they interrelate in old age? Am J Geriatr Psychiatry 21: 398-410.

Vinkers DJ, Gussekloo J, Stek ML, Westendorp RGJ, van der Mast RC. 2004. Temporal relation between depression and cognitive impairment in old age: prospective population based study. BMJ 329.

Wilson RS, Mendes DCF, Bennett DA, Beinias J, Evans D. 2004. Depressive symptoms and cognitive decline in a community population of older persons. J Neurol Neurosurg Psychiatry 75: 126-129. 